\title{
An Improved Simultaneous-Revelation Resolution Procedure that Induces Truthfulness
}

\author{
Linlan Zhang ${ }^{1, *}$, Chunmei $\mathrm{Wu}^{2}$, Zhongyan Wang ${ }^{2}$, Zefang $\mathrm{Li}^{3}$ \\ ${ }^{1}$ Institute of Systems Engineering, Huazhong University of Science and Technology, Wuhan, Hubei, 430074, China; ${ }^{2}$ Department of \\ the Basics, Air Force Radar Academy, Wuhan, Hubei, 430019, China; ${ }^{3}$ Institute of Computer Technology, Wuhan Institute of Tech- \\ nology, Wuhan, Hubei, 430047, China. \\ E-mail: zhanglinlan@smail.hust.edu.cn
}

Received February $2^{\text {nd }}, 2009$; revised May $25^{\text {th }}, 2009$; accepted April $20^{\text {th }}, 2009$.

\begin{abstract}
Alternating-offer bargaining protocol is the most predominant way for solving bilateral bargaining problem in daily life. However, alternating-offer consumed more time and caused a lower efficiency in some cases. One proposed solution is called simultaneous-revelation resolution by which both parties reveal their reservation prices at the same time. But most simultaneous-revelation resolution procedures are inefficient because they encourage exaggerations. But it is fast and uncomplicated, this resolution procedure still has merit-especially if the parties can refrain from undue exaggeration. The paper designs a truthful mechanism for simultaneous-offer bargaining negotiation. In this mechanism, a rule manipulator can induce buyer and seller both to reveal their real prices by introducing suitable adjustment functions. And we show the honest revelations are in Nash equilibrium.
\end{abstract}

Keywords: mechanism design, simultaneous-offer, bargaining, truthfulness, nash equilibrium

\section{Introduction}

Agent mediated negotiation has received considerable attention in the field of electronic commerce [1,2]. The simplest form of negotiation involves two agents and a single-issue. Negotiation is a process that allows disputing agents to decide how to divide the gains from cooperation [3,4]. The face-to-face, open-ended bargaining is the most commonly used way for solving the problems. Bargaining is normally studied using either the axiomatic approach introduced by Nash [5], or the strategic approach, for which Rubinstein's [6] alternating offer model is probably the most influential [7]. However, alternating-offer consumed more time and caused a lower efficiency in some cases. Informal bargaining, without any imposed structure for negotiations and without tight time constrains, leads to more efficient outcomes than do most formal methods. One proposed structured alternative to informal bargaining is the procedure by which both parties reveal their reservation prices at the same time. If the buyer's bid is at least as large as the seller's ask, then the item is sold at a price between the two offers and the agreement will be settled.

"Corresponding author, Tel.: +86 13260647160, Fax: +86 2765692068.
The conflicts between parties are resolved and the parties' payoffs both are better off [8]. If the offers do not overlap, then no trade takes place and the negotiations are broken off.

This mechanism was first modeled by Chatterjee \& Samuelson [9], and then studied in more details by Myerson and Satterthwaite [10], Leininger et al. [11]. This alternative, though appealing, does not work very well [12]. According to a commonly proposed symmetric resolution procedure, the parties simultaneously submit their reservation prices to the mediator. Let these disclosed values be $s^{\prime}$ (not necessarily the true reservation price $s$ ) for the seller, and $b^{\prime}$ (not necessarily the true reservation price $b$ ) for the buyer. If $b^{\prime}<s^{\prime}$, then the negotiation is broken off ; If $s<b$, the final contract will be $x^{*}=\left(b^{\prime}+s^{\prime}\right) / 2$, the midpoint between $b^{\prime}$ and $s^{\prime}$ (see Figure 1).

When this simultaneous-revelation resolution procedure was tried, most parties gave truthful revelations: $s^{\prime}$ equaled $s$, and $b^{\prime}$ equaled $b$ [12]. However, in some cases $s^{\prime}$ was greater than $s$ (because the seller want the midpoint drift right), and $b^{\prime}$ less than $b$ (because the buyer want the midpoint drift left); indeed, in some of 


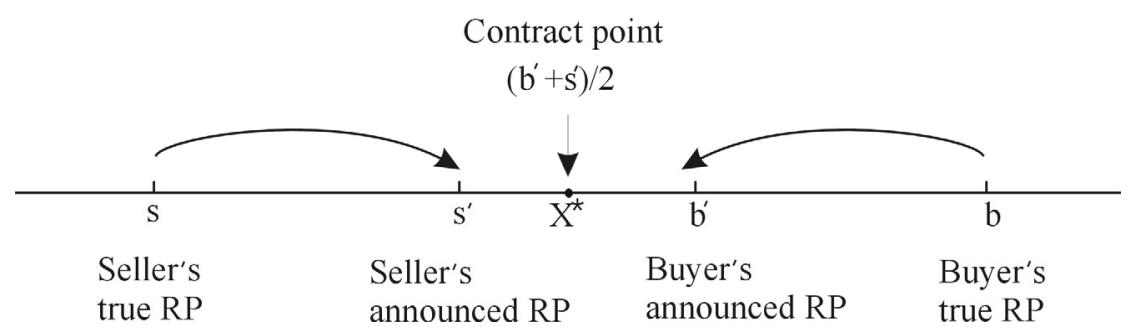

Figure 1. The simultaneous revelation produce

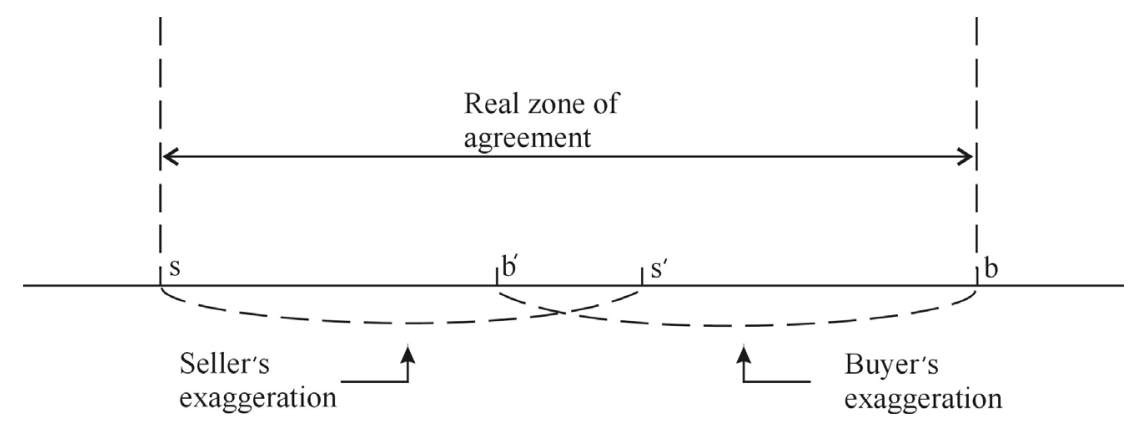

Figure 2. Case in which there is a Zone of Agreement in real but not in revealed values

these cases, there was in fact a zone of agreement ( $s$ was less than $b$ ) but the parties did not detect it $\left(s^{\prime}\right.$ was greater than $\left.b^{\prime}\right)$ and an inefficiency resulted. If both parties exaggerate a lot, then the chances for an agreement are very poor (see Figure 2).

Thus, the simultaneous-revelation resolution procedure is inefficient because it encourages exaggerations; but it's fast and uncomplicated. If time is at a premium or if one is engaged in many such bargaining problems, then this resolution procedure still has merit -especially if the parties can refrain from undue exaggeration. Perhaps the most interesting questions are those that game theoretic ones. Possibly the most central problem of this form is the difficulty of getting efficient truthful mechanisms. The basic game-theoretic requirement in mechanism design is that of "truthfulness" (incentive compatibility), i.e. that each participating agent is motivated to cooperate with the protocol and to report his true valuation [13].

This paper gives an improved simultaneous-revelation resolution procedure that can engender truthfulness. In this mechanism, rule manipulator can induce buyer and seller both reveal their real prices with suitable adjustment functions. And we show honest revelations are in Nash equilibrium [14].

The rest of this paper is structured as follows. In Section 2 we formally present our model and notations. In
Section 3 we show three propositions about honestly revelation. Section 4 concludes.

\section{Negotiation Model and Discussions}

We formally present our model: the mechanism under consideration, its basic components and the assumptions on the parties.

\subsection{Bargaining Rules}

Suppose that there is a seller $S$, a buyer $B$, and a rules manipulator $M$. Before bargaining starts, $S$ and $B$ must submit their reservation prices and the sincerity prices (price constraints that a trader considers the opponent's offer have to satisfy) to $M$. These valuations are their private information. Let $s_{1}$ and $b_{1}$ denote the reservation price and the sincerity price of $S$ respectively; Let $b_{2}$ and $s_{2}$ denote the reservation price and the sincerity price of $B$ respectively. They must satisfy the relation $b_{1} \leq s_{1} \leq b_{2} \leq s_{2}$. Let $S$ and $b$ denote the offer of $S$ and $B$ respectively. $M$ stipulates: $s$ and $b$ must satisfy $b_{1} \leq b \leq b_{2}$ and $s_{1} \leq s \leq s_{2}$; otherwise considers they does not have the sincerity and does not permit the transaction. If $s \leq b$, then the final contract will be the midpoint $x^{*}=(b+s) / 2$. In addition, both the parties may obtain an adjusted amount that the opponent will 
pay them that depends on the price they announce. If $b \leq s$, they can't get anything from disagreement.

\subsection{Assumptions}

It's important to keep in mind that the parties must agree to the payoff procedure before they begin to bargain. The adjusted amount is extracted according to following adjusted function respectively. We express the adjusted function of $S$ and $B$ with $f(s)$ and $g(b)$ respectively as follows:

$$
\begin{aligned}
& f(s)= \begin{cases}\left(b_{2}-b\right)(b-s) /\left(2\left(b_{2}-b+s-s_{1}\right)\right), & s \neq s_{1} \\
\left(b_{2}-s_{1}\right) / 2 & s=s_{1}\end{cases} \\
& g(b)= \begin{cases}\left(s-s_{1}\right)(b-s) /\left(2\left(b_{2}-b+s-s_{1}\right)\right), & b \neq b_{2} \\
\left(b_{2}-s_{1}\right) / 2 & b=b_{2}\end{cases}
\end{aligned}
$$

Notice that the number $s$ is bigger, the function value of $f(s)$ is smaller, namely the higher $s$ the lower the adjusted payment $S$ will receive from $B$. Similarly, the number $b$ is smaller, the function value of $g(b)$ is smaller, namely the lower $b$ the lower the adjusted payment $B$ will receive from $S$. Hence, there is less incentive for $S$ to exaggerate and for $B$ to lessen with the adjustment. We will prove the adjusted functions can induce $S$ and $B$ to make honest revelations: $s=s_{1}, b=b_{2}$, and honest revelations are in Nash equilibrium. All this assumes that they are trying to maximize their utility and they both are risk neutral.

\subsection{Main Results}

According to the rule, we know that the utility function of seller $\mathrm{S}$ and buyer $\mathrm{B}$ respectively is:

$$
\begin{aligned}
& U_{s}=(b+s) / 2-s_{1}+f(s)-g(b), \\
& U_{B}=b_{2}-(b+s) / 2+g(b)-f(s) .
\end{aligned}
$$

We could obtain the following proposition.

Proposition 1. No matter the buyer whether to offer honestly, $s=s_{1}$ is optimal for the seller.

Proof. Considering the following two cases:

1) $b \neq b_{2}:$ in this case, if $s=s_{1}$, we have

$U_{S 1}=\left(b+s_{1}\right) / 2-s_{1}+\left(b_{2}-s_{1}\right) / 2=\left(b+b_{2}\right) / 2-s_{1}$.

If $s \neq s_{1}$, we have

$$
\begin{aligned}
U_{s 2} & =(b+s) / 2-s_{1}+(b-s) / 2-\left(s-s_{1}\right)(b-s) /\left(b_{2}-b+s-s_{1}\right) \\
& =b-s_{1}-\left(s-s_{1}\right)(b-s) /\left(b_{2}-b+s-s_{1}\right) .
\end{aligned}
$$

From the relation $s_{1}<s \leq b<b_{2}$, we have

$$
\left(s-s_{1}\right)(b-s) /\left(b_{2}-b+s-s_{1}\right) \geq 0 .
$$

Then we have $U_{s 2} \leq b-s_{1}<\left(b+b_{2}\right) / 2-s_{1}$, i.e., $U_{S 2}<U_{S 1}$. Therefore, against $b \neq b_{2}$ the optimum response of seller $S$ is $s=s_{1}$.

2) $b=b_{2}:$ in this case, if $s=s_{1}$, we have

$$
\bar{U}_{S 1}=\left(b_{2}+s_{1}\right) / 2-s_{1}=\left(b_{2}-s_{1}\right) / 2 \text {. }
$$

If $s \neq s_{1}$, we have

$$
\bar{U}_{s 2}=\left(b_{2}+s\right) / 2-s_{1}-\left(b_{2}-s_{1}\right) / 2=\left(s-s_{1}\right) / 2 \text {. }
$$

According to the relation $s \leq b_{2}$, we have $\left(s-s_{1}\right) / 2 \leq$ $\left(b_{2}-s_{1}\right) / 2$, i.e., $\bar{U}_{s 2} \leq \bar{U}_{s 1}$. Therefore, against $b=b_{2}$ the optimum response of seller $S$ is $\quad s=s_{1}$.

Thus, no matter buyer B whether to offer honestly, $s=s_{1}$ is optimal for seller $S$.

Proposition 2. No matter the seller whether to offer honestly, $b=b_{2}$ is optimal for the buyer.

Proof. Considering the following two cases:

1) $s \neq s_{1}$ : in this case, if $b=b_{2}$, we have

$$
\begin{aligned}
U_{B 1} & =b_{2}-\left(b_{2}+s\right) / 2+\left(b_{2}-s_{1}\right) / 2 \\
& =b_{2}-\left(s+s_{1}\right) / 2 .
\end{aligned}
$$

If $b \neq b_{2}$, we have

$$
\begin{aligned}
U_{B 2} & =b_{2}-\frac{b+s}{2}+\frac{b-s}{2}-\frac{\left(b_{2}-b\right)(b-s)}{b_{2}-b+s-s_{1}} \\
& =b_{2}-s-\frac{\left(b_{2}-b\right)(b-s)}{b_{2}-b+s-s_{1}} .
\end{aligned}
$$

According to the relation $s_{1}<s \leq b<b_{2}$, we have $\left(b_{2}-b\right)(b-s) /\left(b_{2}-b+s-s_{1}\right) \geq 0$.

Then we have $U_{B 2} \leq b_{2}-s<b_{2}-\left(s+s_{1}\right) / 2$, i.e., $U_{B 2}<U_{B 1}$. Thus against $s \neq s_{1}$ the optimum response of buyer $\mathrm{B}$ is $b=b_{2}$.

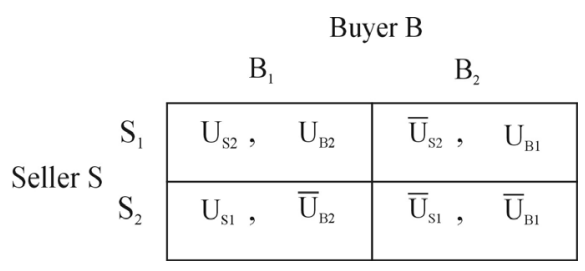

Figure 3. The strategic form game of the buyer and the seller 
2) $s=s_{1}$ : in this case, if $b=b_{2}$, there is

$$
\bar{U}_{B 1}=b_{2}-\left(b_{2}+s_{1}\right) / 2=\left(b_{2}-s_{1}\right) / 2 \text {. }
$$

If $b \neq b_{2}$, there is

$$
\bar{U}_{B 2}=b_{2}-\left(b+s_{1}\right) / 2-\left(b_{2}-s_{1}\right) / 2=\left(b_{2}-b\right) / 2 \text {. }
$$

According to the relation $s_{1} \leq b$, we have the relation $\left(b_{2}-b\right) / 2 \leq\left(b_{2}-s_{1}\right) / 2$, i.e., $\bar{U}_{B 2} \leq \bar{U}_{B 1}$. Therefore, against $S=S_{1}$ the optimum response of buyer B is $b=b_{2}$.

Thus, no matter seller $S$ whether to offer honestly, $b=b_{2}$ is optimal for buyer $B$.

Let $S_{1}$ and $S_{2}$ denote two strategies of $S$ : $S_{1}: s \neq S_{1}, S_{2}: s=S_{1}$. Let $B_{1}$ and $B_{2}$ denote two strategies of $\mathrm{B}: B_{1}: b \neq b_{2}, B_{2}: b=b_{2}$. This strategic form game is available from Figure 3. According to the following four inequalities:

$$
U_{S 2}<U_{S 1}, \bar{U}_{S 2} \leq \bar{U}_{S 1}, U_{B 2}<U_{B 1}, \bar{U}_{B 2} \leq \bar{U}_{B 1},
$$

We could obtain the following proposition.

Proposition 3. Strategy combination $\left(S_{2}, B_{2}\right)$ forms a Nash equilibrium, and in this case, $U_{S}=U_{B}=$ $\left(b_{2}+s_{1}\right) / 2$, the net side payment of the seller and the buyer both are zero, i.e., $f(s)-g(b)=0$.

Proof. The first half part is obvious, we prove the second half. When $s=s_{1}$ and $b=b_{2}$, we have $f(s)=g(b)=\left(b_{2}-s_{1}\right) / 2$. Then we have $f(s)-g(b)$ $=0$, and

$$
\begin{aligned}
& U_{s}=(b+s) / 2-s_{1}+f(s)-g(b)=\left(b_{2}-s_{1}\right) / 2, \\
& U_{B}=b_{2}-(b+s) / 2+g(b)-f(s)=\left(b_{2}-s_{1}\right) / 2 .
\end{aligned}
$$

i.e., $U_{S}=U_{B}=\left(b_{2}+s_{1}\right) / 2$. The proof is completed.

\section{Conclusions}

In this study, our results have important constructions for procedure design of a simultaneous-offer bargaining system that can engender truthfulness. But in order to implement this scheme, the seller and the buyer have to approve of the rules manipulator before they start the agreement. It is rather restrictive, but the result is so appealing that it should not be lightly dismissed. With suitable adjustment functions, honest revelations are in Nash equilibrium: each party should tell the truth if the other does. It would be wonderful if someone could apply this scheme to real-world situations. This is also the direction we will study diligently in the future.

\section{REFERENCES}

[1] R. P. Maes and A. G. Moukas, "Agents that buy and sell," Communications of the ACM, Vol. 42, No. 3, pp. 81-91, 1991.

[2] T. Sandholm, "Agents in electronic commerce: Component technologies for automated negotiation and coalition formation," Autonomous Agents and Multi-Agent Systems, Vol. 3, No. 1, pp. 73-96, 2000.

[3] J. S. Rosenschein and G. Zlotkin, "Rules of encounter," The MIT Press, 1994.

[4] S. Kraus, "Strategic negotiation in multi-agent environments," Cambridge: The MIT Press, Massachusetts, 2001.

[5] J. Nash, "The bargaining problem," Econometrica, No. 18, pp. 155-162, 1950

[6] A. Rubinstein, "Perfect equilibrium in a bargaining model," Econometrica, No. 50, pp. 97-110, 1982.

[7] G. J. Grenier, et al., "Sociological theory," B. SYA6126, Spring 2007.

[8] Y. J. Hu, "Trusted agent-mediated E-commerce transaction services via digital certificate management," Electronic Commerce Research, Vol. 3, pp. 221-243, 2003.

[9] K. Chatterjee and W. Samuelson, "Bargaining under incomplete information," Operations Research, No. 31, pp. 835-851, 1983.

[10] R. B. Myerson and M. A. Satterthwaite, "Efficient mechanisms for bilateral trading," Journal of Economic Theory, No. 29, pp. 265-281, 1983.

[11] W. Leininger, P. B. Linhart, and R. Radner, "Equilibria of the sealed-bid mechanism for bargaining with incomplete information," Journal of Economic Theory, Vol. 48, pp. 63-106, 1989.

[12] H. Raiffa, "The art and science of negotiation," Cambridge: Harvard University Press, MA, 1982.

[13] A. Mu'alem and N. Nisan, "Truthful approximation mechanisms for restricted combinatorial auctions," Games and Economic Behavior, Doi: 10.1016/j.geb.2007. 12.009, 2008.

[14] Y. F. Luo, "Game theory course," Peking: Tsinghua University Press, Peking Jiaotong University Press, 2007. 